\title{
WORKSPACE ANALYSIS AND OPTIMIZATION OF THE PARALLEL ROBOTS BASED ON COMPUTER-AIDED DESIGN APPROACH
}

\author{
Badreddine Aboulissane, Larbi EI Bakkali, Jalal El Bahaoui
}

Team Modeling and Simulation of Mechanical Systems, Faculty of Sciences, Abdelmalek Essaadi University, Tetouan, Morocco

\begin{abstract}
This paper provides workspace determination and analysis based on the graphical technique of both spatial and planar parallel manipulators. The computation and analysis of workspaces will be carried out using the parameterization and threedimensional representation of the workspace. This technique is implemented in CAD (Computer Aided Design) Software CATIA workbenches. In order to determine the workspace of the proposed manipulators, the reachable region by each kinematic chain is created as a volume/area; afterwards, the full reachable workspace is obtained by the application of a Boolean intersection function on the previously generated volumes/areas. Finally, the relations between the total workspace and the design parameters are simulated, and the Product Engineering Optimizer workbench is used to optimize the design variables in order to obtain a maximized workspace volume. Simulated annealing $(S A)$ and Conjugate Gradient $(C G)$ are considered in this study as optimization tools.
\end{abstract}

Key Words: CAD Software, Design Parameters, Optimization, Parallel Robots, Workspace Analysis

\section{INTRODUCTION}

A parallel manipulator is a mechanism in which there are two or more closed kinematic chains attaching the base to the mobile platform. Nowadays, most of the manipulators are serial architecture; parallel robots exhibit many advantages, such as high speeds and accelerations, low mobile masses, high stiffness, and great accuracy. The most notable disadvantage of the parallel manipulators is their relatively small workspaces. One can use the workspace volume or surface as an objective function for optimization. In this sense, the researchers focused on workspace determination as a performance index in order to design robots for specific industrial applications. The calculation of the parallel robots' workspace

Received April 28, 2019 / Accepted January 25, 2020

Corresponding author: Badreddine Aboulissane

Team Modeling and Simulation of Mechanical Systems, Faculty of Sciences, Abdelmalek Essaadi University, BP. 2121 M'Hannech II, Tetouan, Morocco

E-mail: b.aboulissane@gmail.com 
is a complex problem due to the kinematic modeling difficulty. However, the problem of workspace optimization of the parallel manipulators to obtain a prescribed workspace has been investigated in few articles. The concept of the prescribed workspace is a significant issue to optimize and to synthesize a robot. The actuated joint variables, the range of joints motion and the mechanical interferences between the links essentially influence the parallel manipulators' workspace. In this paper, we focus on some areas of the space that surrounds the manipulator, and limiting its workspace to the prescribed area. Several papers studied this problem based on geometrical techniques, and using optimization algorithms to synthesize the design parameters of the parallel manipulators.

Gallant and Boudreau [1] used a Genetic Algorithm in order to optimize a 3-DOF planar parallel manipulator to obtain a workspace as close as possible to a prescribed one. Singularities and workspace of planar 3-RPR parallel mechanism for maximal singularityfree workspace by optimizing the geometric parameters are investigated by Jiang and Gosselin [2, 3] and Yang and O'Brien [4]. Di Gregorio and Zanforlin [5] studied the workspace of the 3-RUU and the DELTA robot. They concluded that these robots could have the same closure equations and workspace when some geometric conditions are satisfied. Chablat et al. [6, 7] compared 3-DOF parallel kinematic machines using two design criteria: regular workspace shape and a kinetostatic performance index that needs to be as homogenous as possible throughout the workspace ; this technique is based on the interval analysis method. In [8] the workspace optimization of translational 3-UPU parallel robot is performed using its parameterization by two design variables, which are the prismatic joint stroke and the distance between the base and the mobile platform. Zhao, $\mathrm{Chu}$, and Feng [9] discussed the analogous symmetry properties between the workspace and the mechanism structure. Gao, Liu, and Chen [10] analyzed the relationship between the shapes of the workspaces and the link lengths for 3-DOF planar parallel manipulators; his results are useful for the designers to optimize the robots regarding the workspace index. Hay and Snyman $[11,12]$ focused on the numerical multi-level optimization for the synthesis of the 3-DOF parallel manipulators for a desired workspace. In [13] the workspace of Gough-Stewart platform was optimized using the Genetic Algorithm. The idea is to minimize the areas, which do not belong to the intersection between two areas: the workspace of the robot and the prescribed workspace. A genetic algorithm based method is used also in [14] to deal with the optimal dimensional synthesis of the DELTA robot for a prescribed workspace. The geometrical approaches have been used to represent the workspace of the parallel manipulators, by Assad Arrouk et al. [15], Aboulissane et al. [21], Bonevet al. [16], Gosselin [17], and Merlet [18]. The principle of these methods is to deduce, from the constraints on each limb, a geometrical entity (sub-workspace) which describes all the possible poses of the tool center point that satisfy the leg constraints. Then, the robotic manipulator workspace is generated by the intersection of all the subworkspaces. Tsirogiannis et al. [22] presented an overall structural design optimization approach for a robot arm link seeking mass reduction and satisfaction of manufacturability with SLS AM technique.

In this paper, a graphical based technique is addressed for workspace's determination, analysis and optimization of two parallel robots, which are the 3-RPR planar manipulator, and the DELTA robot.

The first section is dedicated to the description of the proposed manipulators. In the next section, we present the steps to determine the workspace of both robots. The last section is about the comparison of the two optimization methods, applied to the workspace of the DELTA robot. 


\section{KINEMATIC SCHEME AND DESCRIPTION OF THE 3-RPR AND THE DELTA ROBOTS}

\subsection{The 3-RPR Planar Parallel Robot}

Fig. 1 shows the Kinematic scheme of the 3-RPR planar robot. This mechanism is a parallel robot with closed loop chains. Three actuated prismatic joints are linked to passive joints $A_{1}, A_{2}$, and $A_{3}$ fixed to the base, and $B_{1}, B_{2}, B_{3}$ fixed to the mobile platform. The actuated prismatic joints coordinates are given by the length of the legs, named $\rho_{1}, \rho_{2}$, and $\rho_{3}$. The orientation of the mobile platform is given by angle $\varphi$. The components of points $A_{i}$ and $B_{i}$ are respectively $\left(x_{a i}, y_{a i}\right)$ and $\left(x_{b i}, y_{b i}\right)$. Each limb generates an annular region bounded by two concentric circles with radii of $\rho_{i, \min }$ and $\rho_{i, \max }$, the centers of the circles $C_{i}\left(x_{c i}, y_{c i}\right)$ are defined by Eq. (1), and Eq. (2):

$$
\begin{aligned}
& x_{c i}=x_{a i}-x_{b i} \cos (\varphi)+y_{b i} \sin (\varphi) \\
& y_{c i}=y_{a i}-x_{b i} \sin (\varphi)-y_{b i} \cos (\varphi)
\end{aligned}
$$

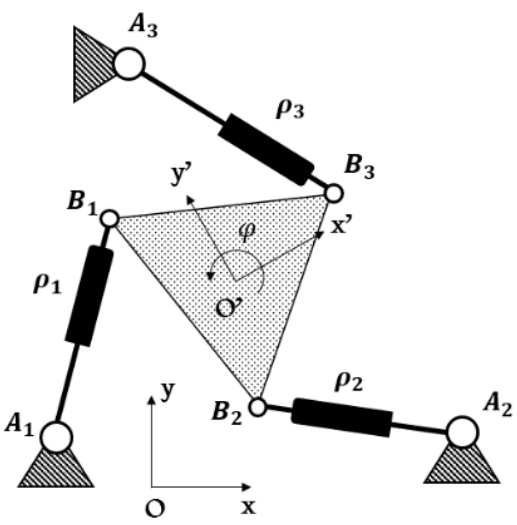

Fig. 1 Kinematic diagram of the 3-RPR planar parallel robot

The vector describing the 3-RPR parallel manipulator parameters is defined as follows:

$$
\delta_{1}=\left[\rho_{\min } \rho_{\max } x_{c 1} y_{c 1} x_{c 2} y_{c 2} x_{c 3} y_{c 3}\right]^{T}
$$

\subsection{The DELTA Parallel Robot}

The robot under study in this section is the DELTA parallel robot depicted in Fig. 2(a); it is composed of a triangular moving platform linked to a triangular fixed base with three closed parallel chains. Each one consists of an actuated rotational joint mounted near to the fixed base; the parallelograms and spherical joints transmit the movement of the mobile platform. In this work, all the three arms of the manipulator are identical in terms of geometrical parameters (Fig. 2(b)). 


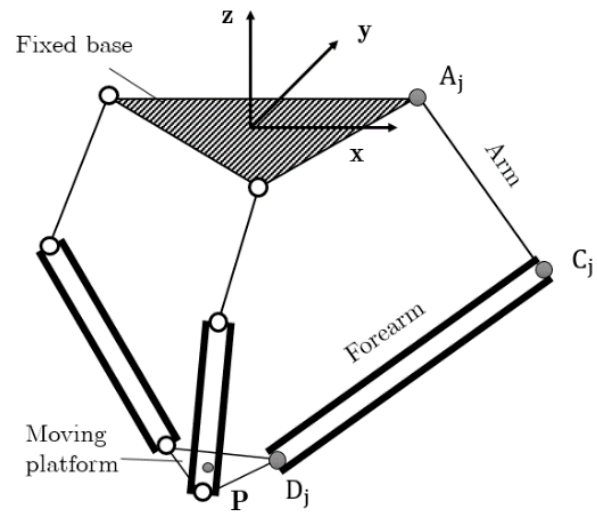

(a)

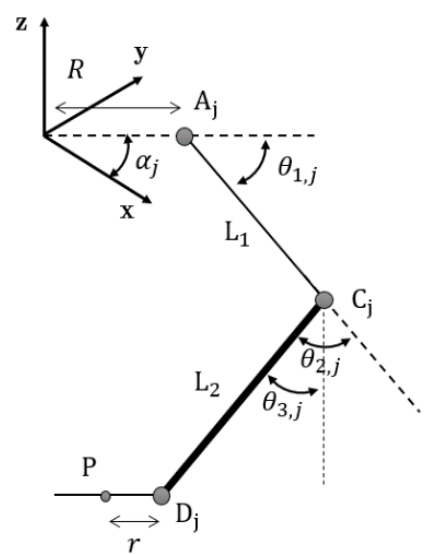

(b)

Fig. 2 (a) Geometric scheme of the Delta robot; (b) the DELTA robot parameterization

The independent design variables for the DELTA robot are:

$$
\delta_{2}=\left[\begin{array}{llll}
L_{1} & L_{2} & R & r
\end{array}\right]^{T}
$$

\section{WORKSPACE DETERMINATION OF THE PROPOSED ROBOTS}

First, we need to determine workspace of the proposed robots. We can define this region as the reachable positions and rotations by the end-effector center point, generally located on the platform of the robots. In this work, we used a geometrical technique for the representation of the workspace through the CATIA software, and there are no limits for all the revolute joints used for each manipulator.

\subsection{Workspace of the 3-RPR Planar Robot}

For this robot, we obtain the workspace by the intersection of three circular areas, which correspond to the areas accessible by the end-effector point center when each leg is taken as a serial manipulator. Fig. 3 shows the steps we followed to generate the workspace of the 3RPR manipulator in the CATIA.

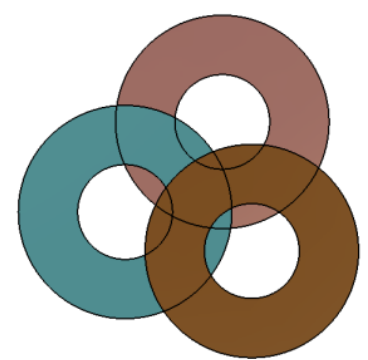

(a)

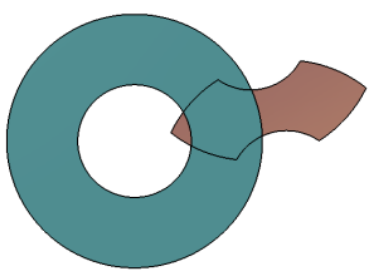

(b)

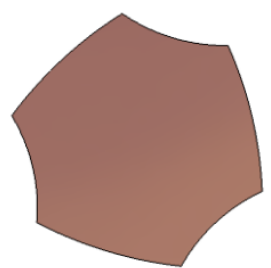

(c)

Fig. 3 Steps of the workspace determination of the 3-R $\underline{P R}$ robot[15] 
Fig. 3(a) depicts the first step; it consists of creating three annular regions in the Sketcher workbench; then the Pad and Pocket commands are applied to the drawing with a finite thickness in the Part Design workbench. The second step is performed under the Part Design workbench; it consists of applying the first intersection Boolean operation. Fig. 3(b) above shows the obtained result.

The final step to determine the workspace of the 3-RPR is to apply a second intersection Boolean operation on the two remaining regions in the second step. The obtained shape corresponds to the theoretical workspace of the mechanism shown in Fig. 3(c). This step is also done in the Design Part workbench.

The area of the 3D model presented in Fig. 3(c) is calculated by using a smart area parameter. Since the thickness of this 3D model is neglected, the workspace area of the 3$\mathrm{R}$ PR robot is obtained, dividing by two, the area previously calculated.

The design parameters used to obtain this workspace are tabulated in Table 1:

Table 1 Design parameters of the 3-R $\underline{P R}$ manipulator

\begin{tabular}{lrrr}
\hline Design parameters & Limb 1 & Limb 2 & \multicolumn{1}{c}{ Limb 3 } \\
\hline$x_{c i}(\mathrm{~mm})$ & $-188,632$ & 132,159 & 56,473 \\
$y_{c i}(\mathrm{~mm})$ & $-43,697$ & $-141,512$ & 185,209 \\
\hline$\rho_{\min }(\mathrm{mm})$ & & 120 & \\
$\rho_{\max }(\mathrm{mm})$ & & 270 & \\
$\varphi($ degrees $)$ & & 102 & \\
Area $\left(\mathrm{cm}^{2}\right)$ & & 197,02 & \\
\hline
\end{tabular}

For each orientation $\varphi$, the workspace of the robot has different shape and area. Table 2 illustrates the workspace of the robot for few orientations of the mobile platform.

Table 2 Variation of the workspace with respect to the orientation of the mobile platform

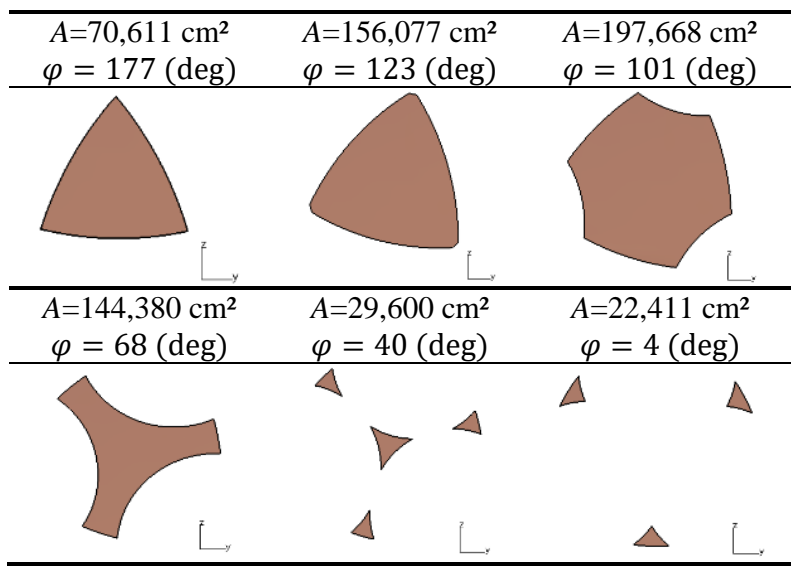

\subsection{Workspace of the DELTA robot}

The workspace of the DELTA Parallel robot is defined as a three dimensional volume in the Cartesian space; this volume is reached by a point on the mobile platform. The 
equations used to determine the workspace of a parallel robot are generally complex to solve by using the traditional approaches. Hence, the CAD-based approach is used in this work to determine geometrically the workspace of the DELTA parallel robot.

The parallel robot workspace robot can be quickly generated as an area or a volume using the CATIA, then the complex technique such the numerical method. Discretization based techniques produce an approximate form of a low quality workspace. To improve it, it is necessary to use other graphical methods. By implanting the problem of workspace determination in a CAD software, these techniques will become more reachable to industry and more precise. As the first step, the proposed method for workspace determination of the DELTA robot consists in assuming all legs to be independent serial arms having the mobile platform as tool center point. Then, the region swept by the tool center point of each arm is determined for a given orientation of this point.

In [14] the workspace of the DELTA robot is presented by following Eq. (5):

$$
\left[\left(x_{t}-\Delta r\right)^{2}+y_{t}^{2}+z_{t}^{2}+L_{1}^{2}-L_{2}^{2}\right]^{2}=4 L_{1}^{2}\left[\left(x_{t}-\Delta r\right)^{2}+z_{t}^{2}\right]
$$

with $\Delta r=R-r$ and:

$$
\left\{\begin{array}{l}
x_{t}=x \cos \alpha_{i}+y \sin \alpha_{i} \\
y_{t}=-x \sin \alpha_{i}+y \cos \alpha_{i} \\
z_{t}=z
\end{array}\right.
$$

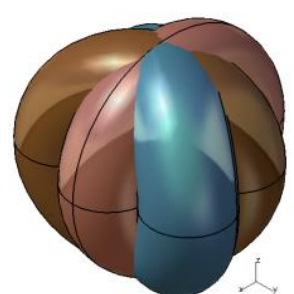

(a)

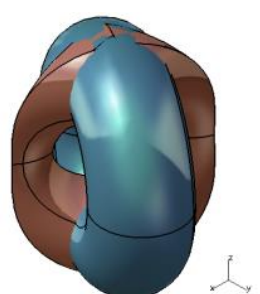

(b)

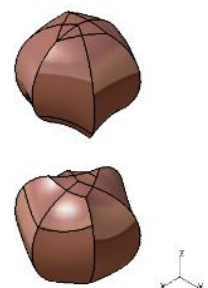

(c)

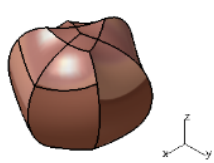

(d)

Fig. 4 Generation of the workspace for a Delta robot based on the CATIA V5. (a) The three torus intersect; (b) First intersection Boolean operation; (c) Second Intersection; (d) The workspace of the Delta robot $(\mathrm{z}<0)$

As shown in Fig. 4, the workspace of the Delta robot is based on three tori. The first step consists of drawing a circle with a radius $L_{1}$, and another circle with a radius $L_{2}$ passing by the center of the first circle (Fig. 5).

Each limb of the DELTA robot generates a torus, Fig.4(a) depicts the intersection of those three volumes, and the rest of the figures shows the intersection Boolean operations with the final shape of the workspace of the robot presented by Fig. 4(d).

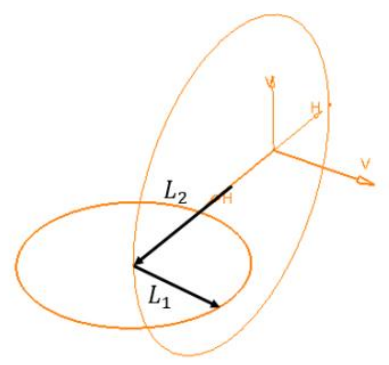

Fig. 5 Torus in Sketcher workbench 


\subsection{Workspace analysis of the DELTA robot}

Before starting the optimization problem, an analysis between the reachable workspace and the design parameters is required. We presented examples for each variable $L_{1}$ and $L_{2}$, as well as the resulting volume. First we fixed $R=55 \mathrm{~mm}, r=30 \mathrm{~mm}$, and $L_{1}=100 \mathrm{~mm}$, then length $L_{2}$ is varied from $100 \mathrm{~mm}$ to $250 \mathrm{~mm}$. Table 3 shows the boundaries of the reachable workspace for length $L_{2}$ of $100 \mathrm{~mm}, 150 \mathrm{~mm}, 200 \mathrm{~mm}$, and $250 \mathrm{~mm}$.

Table 3 Workspace shape versus $\boldsymbol{L}_{2}$

\begin{tabular}{cccc}
$L_{2}=100 \mathrm{~mm}$ & $\begin{array}{c}L_{2}=150 \mathrm{~mm} \\
W=4,688 \mathrm{dm}^{3}\end{array}$ & $\begin{array}{c}L_{2}=200 \mathrm{~mm} \\
W=4,765 \mathrm{dm}^{3}\end{array}$ & $\begin{array}{c}L_{2}=250 \mathrm{~mm} \\
W=4,647 \mathrm{dm}^{3}\end{array}$ \\
\hline
\end{tabular}

It can be seen from Table 3 that the workspace volume of the DELTA robot increases with reducing length $L_{2}$ of the forearm.

Now, to demonstrate the effect of length $L_{1}$, forearm $L_{2}$ is fixed at $250 \mathrm{~mm}, R=55 \mathrm{~mm}$, $r=30 \mathrm{~mm}$, and $L_{1}$ is varied from $100 \mathrm{~mm}$ to $250 \mathrm{~mm}$.

Table 4 Workspace shape versus $L_{1}$

$\begin{array}{cccc}L_{1}=100 \mathrm{~mm} & L_{1}=150 \mathrm{~mm} \\ W=4,647 \mathrm{dm}^{3} & \begin{array}{c}L_{1}=200 \mathrm{~mm} \\ W=37,637 \mathrm{dm}^{3}\end{array} \\ W=15,753 \mathrm{dm}^{3} & L_{1}=250 \mathrm{~mm} \\ W=75,040 \mathrm{dm}^{3}\end{array}$

As shown in Table 4, the workspace volume increases considerably by increasing the length $L_{1}$. The volume starts to take a cup-shape.

\section{OPTIMIZATION PROBLEM}

In robotics, the designer uses numerous indices to evaluate the performance of a manipulator; among these indices, we can mention the workspace that describes the potential robot utilization. In this work, we are using the reachable workspace as a performance index in order to optimize the design parameters of the DELTA robot. The optimization problem is formulated as follows:

$$
\begin{array}{cc}
\text { maximize } & W\left(\delta_{2}\right) \\
\text { subject to } & \delta_{2, i, \min } \leq \delta_{2, i} \leq \delta_{2, i, \max }
\end{array}
$$


where $W$ is the workspace volume, and $\delta_{2}$ is the vector defined by Eq. (4). The main purpose of the maximization of the workspace is to expand the capabilities of the DELTA robot. The parameters that have an effect on the volume and the shape of the reachable workspace of the manipulator are: $L_{1}, L_{2}, R, r$ and $\alpha_{j}$ with $(\mathrm{j}=1,2,3)$. The parameterization used in the CATIA software is shown in Fig. 6:

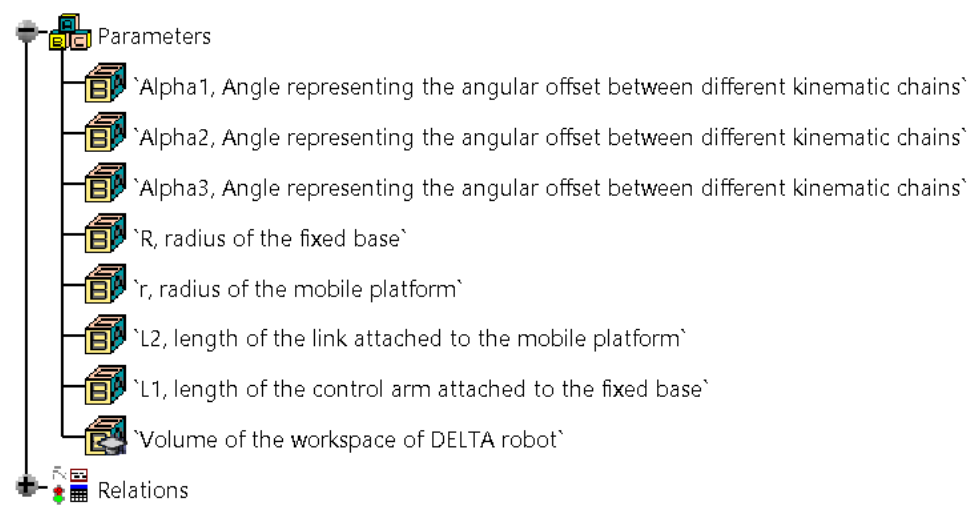

Fig. 6 Parameters of the DELTA robot on the CATIA

For this optimization problem, we used the CATIA "Product Engineering Optimizer" workbench in which we can use different algorithms such as: Conjugate Gradient method (CG) a local algorithm and the simulated annealing (SA) a global algorithm. Both the methods are employed in our present study. The simulated annealing is listed as the oldest algorithm among the metaheuristics that had an explicit strategy to avoid local minima; it can be applied to the majority of optimization problems. The behavior of this algorithm is strongly dependent on the problem addressed [19]. The other algorithm, which is the Conjugate Gradient, is a mathematical approach used on both linear and non-linear systems; this approach can be used as an iterative algorithm and a direct method [20]. To realize this optimization, we choose the last five parameters that are shown in Fig. 6; we excluded the angles $\alpha_{j}$ representing the angular offset between different kinematic chains. The optimization parameters are usually provided with an upper and lower limit. The main goal of this optimization is to maximize the objective function represented by the workspace volume of the DELTA robot, based on the parameters presented in Table 5, which can describe Eq. (7).

The initial parameters correspond to the workspace shown in Table 6(a) with a volume $W=4,765 \mathrm{dm}^{3}$. The first optimization is done using the (SA) algorithm, optimized values are tabulated in Table 5 corresponding to the workspace summarized in Table 6(b) with a volume $W=215,712 \mathrm{dm}^{3}$. Secondly, we applied the (CG) method. Table 6(c) shows the shape of the workspace with a volume $W=110,265 \mathrm{dm}^{3}$. This workspace is associated with the values of the design parameters presented also in Table 5. 
Workspace Analysis and Optimization of the Parallel Robots Based on Computer-Aided Design Approach 87

Table 5 Optimization results

\begin{tabular}{lccc}
\hline Design parameters & Initial values & Simulated annealing & Conjugate gradient \\
\hline$r(\mathrm{~mm})$ & 30 & 193,77 & 54,538 \\
$R(\mathrm{~mm})$ & 55 & 344,198 & 120,308 \\
$L_{l}(\mathrm{~mm})$ & 100 & 350 & 283,757 \\
$L_{2}(\mathrm{~mm})$ & 100 & 112,255 & 250 \\
Volume $\left(\mathrm{dm}^{3}\right)$ & 4,765 & 215,712 & 110,265 \\
\hline
\end{tabular}

Table 6 Workspace optimization

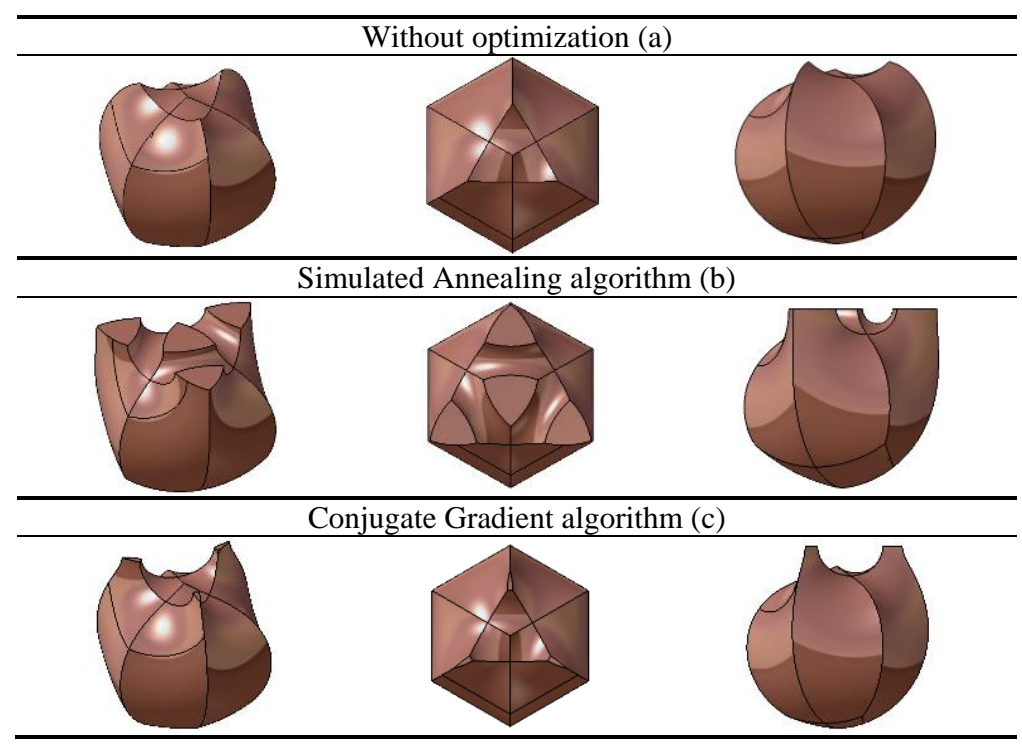

The number of iterations made to reach the objective is 523 for the (SA) algorithm, and 602 for the (CG) method. The time needed to achieve these two optimizations is about 10 minutes. The simulations were performed on a computer that has the following characteristics: CPU @2.10Ghz, 8.0 GB RAM.

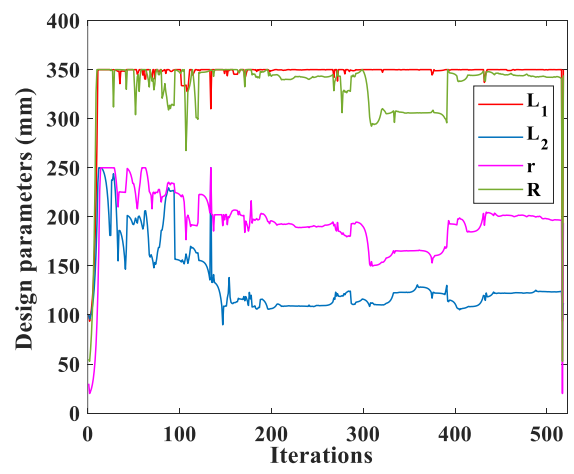

(a)

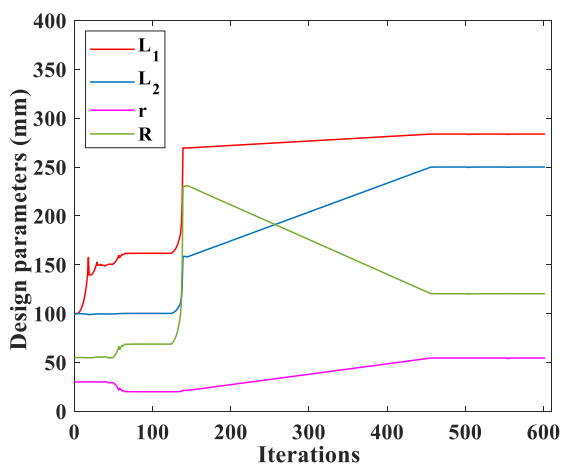

(b)

Fig. 7 Evolution of the design parameters for (a) SA algorithm; (b) CG method 
Fig. 7 presents the evolution of the design variables for both algorithms. From the parametric analysis previously presented in Table 4 and the design variables evolution shown in Figs. 7 (a) and (b), we can conclude that $L_{1}$ have a significant impact on the volume of the workspace. On the other hand, the two algorithms applied in this study have set the $L_{1}$ variable to a maximum value, while other parameters $\mathrm{R}, r$, and $L_{2}$ are showing a variation in a large range searching for a maximum volume of the robot's workspace. Fig. 8 provides a comparison of the convergence rates of the results; it can be seen that the performance of the (SA) is more superior to that of (CG) method due to the good speed of convergence with few generations, also, the optimal value reached by the (SA) algorithm is greater than that obtained by the (GC) algorithm.

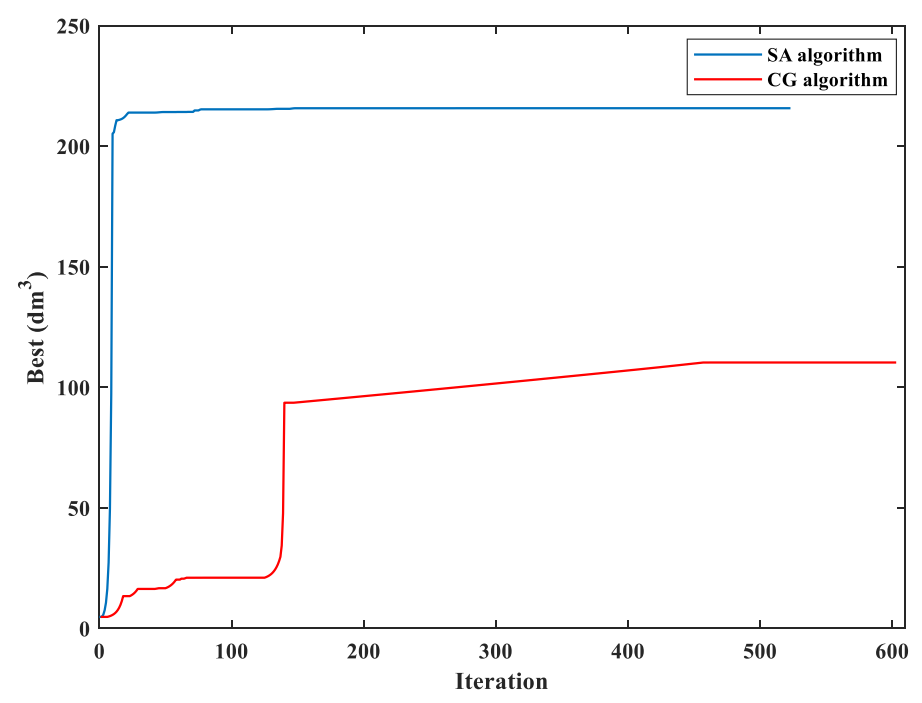

Fig. 8 Comparison of convergence cost for SA and GC algorithms

\section{CONCLUSION}

In this paper, we have focused on the CAD based technique to determine the workspace of planar and spatial parallel robots. This study is performed on the 3-RPR planar parallel robot and the DELTA robot. We considered the determination and the characterization of the workspace of the two manipulators. For this purpose, we have applied a geometrical approach that has been implemented in CATIA workbenches. We put in evidence the effectiveness of this technique for the workspace optimization of the DELTA robot, taking into consideration the joint limits. Two algorithms were applied to maximize the workspace of the DELTA robot, the simulated annealing and the Conjugate Gradient algorithms. The best result is related to the (SA) algorithm in terms of convergence speed and the best optimal value of the workspace volume. The manipulators studied herein for the workspace analysis and optimization illustrate the efficiency and the capability of the graphical methodology for the designers, to avoid complex mathematical equations. 


\section{REFERENCES}

1. Gallant, M., Boudreau, R., 2002, The synthesis of planar parallel manipulators with prismatic joints for an optimal, singularity-free workspace, Journal of Robotic Systems, 19(1), pp. 13-24.

2. Jiang, Q., Gosselin, C.M., 2006, The maximal singularity-free workspace of planar 3-RPR parallel mechanisms, International Conference on Mechatronics and Automation, pp. 142-146.

3. Jiang, Q., Gosselin, C.M., 2007, Geometric optimization of planar 3-RPR parallel mechanisms, Transactions of the Canadian Society for Mechanical Engineering, 31(4), pp. 457-468.

4. Yang, Y., O’Brien, J.F., 2007, A case study of planar 3-RPR parallel robot singularity free workspace design, International Conference on Mechatronics and Automation, pp. 1834-1838.

5. Di Gregorio, R., Zanforlin, R., 2003, Workspace analytic determination of two similar translational parallel manipulators, Robotica, 21(5), pp. 555-566.

6. Chablat, D., Wenger, P., Majou, F., Merlet, J.P., 2004, An interval analysis based study for the design and the comparison of three-degrees-of-freedom parallel kinematic machines, The International Journal of Robotics Research, 23(6), pp. 615-624.

7. Chablat, D., Wenger, P., Merlet, J.P., 2007, A comparative study between two three-dof parallel kinematic machines using kinetostatic criteria and interval analysis, 11th World Congress on Theory of Machines andMechanisms, Tianjin, April, pp. 1209-1213.

8. Badescu, M., Morman, J.,Mavroidis, C., 2002, Workspace optimization of 3-UPU parallel platforms with joint constraints, In Proceedings 2002 IEEE International Conference on Robotics and Automation, 4, pp. 3678-3683.

9. Zhao, J.S., Chu, F., Feng, Z.J., 2008, Symmetrical characteristics of the workspace for spatial parallel mechanisms with symmetric structure, Mechanism and Machine Theory, 43(4), pp. 427-444.

10. Gao, F., Liu, X.J., Chen, X., 2001, The relationships between the shapes of the workspaces and the link lengths of 3-DOF symmetrical planar parallel manipulators, Mechanism and Machine Theory, 36(2), pp. 205-220.

11. Hay, A.M., Snyman, J.A., 2005, A multi-level optimization methodology for determining the dextrous workspaces of planar parallel manipulators, Structural and Multidisciplinary Optimization, 30(6), pp. 422-427.

12. Hay, A.M., Snyman, J.A., 2006, Optimal synthesis for a continuous prescribed dexterity interval of a 3-dof parallel planar manipulator for different prescribed output workspaces, International journal for numerical methods in engineering, 68(1), pp. 1-12.

13. Boudreau, R., Gosselin, C.M., 1999, The synthesis of planar parallel manipulators with a genetic algorithm, Journal of mechanical design, 121(4), pp. 533-537.

14. Laribi, M.A., Romdhane, L., Zeghloul, S., 2008, Advanced synthesis of the DELTA parallel robot for a specified workspace, In Parallel Manipulators, Towards New Applications, Intech Open.

15. Assad, K.A., Bouzgarrou, B.C., Stan, S.D., Gogu, G., 2010, CAD based design optimization of planar parallel manipulators, Diffusion and defect data, Solid state data, Part B, Solid state phenomena, 166, pp. 33-38.

16. Bonev, I.A., Ryu, J., 2001, A geometrical method for computing the constant-orientation workspace of 6PRRS parallel manipulators, Mechanism and machine theory, 36(1), pp. 1-13.

17. Gosselin, C., 1990, Determination of the workspace of 6-DOF parallel manipulators, Journal of mechanical design, 112(3), pp. 331-336.

18. Merlet, J.P., 1995, Determination of the orientation workspace of parallel manipulators, Journal of intelligent and robotic systems, 13(2), pp. 143-160.

19. Kirkpatrick, S., Gelatt, C.D., Vecchi, M.P., 1983, Optimization by simulated annealing, Science, 220(4598), pp. 671-680.

20. Shewchuk, J.R., 1994, An introduction to the conjugate gradient method without the agonizing pain.

21. Aboulissane, B., EL Haiek, D., EL Bakkali, L., EL Bahaoui, J., 2019, On the workspace optimization of parallel robots based on CAD approach, Procedia Manufacturing, 32, pp. 1085-1092.

22. Tsirogiannis, E., Vosniakos, G.C., 2019, Redesign and topology optimization of an industrial robot link for additive manufacturing, Facta Universitatis-Series Mechanical Engineering, 17(3), pp. 415-424. 